\title{
ON THE SYSTEMATIZATION OF THE PROCESS OF MODEL DEVELOPMENT
}

\section{B. LOHMANN and W. MARQUARDT}

\author{
Lehrstuhl für Prozeßtechnik, RWTH Aachen \\ Turmstr. 46, D-52056 Aachen, Germany \\ E-mail: \{lohmann, marquardt\}@lfpt.rwth-aachen.de
}

\begin{abstract}
The lack of adequate support for the development of mathematical process models confines the application of model-based techniques in the design and operation of complex chemical plants. One important issue in addressing this problem is the systematization of the process of model development (called modeling process in the following). In order to provide a basis for further investigations into the modeling process and to prepare the development of computer-aided modeling tools supporting the modeling process, this contribution deals with general properties of the modeling process and discusses to which degree of granularity the modeling process can be formalized. A modeling example will serve to suggest a first set of canonical modeling steps, which allow to classify and continuously extend procedural modeling knowledge.
\end{abstract}

\section{INTRODUCTION}

Due to environmental and safety regulations, growing demands on product quality as well as increasingly competitive markets, the continuous improvement of chemical processes and the development of new ones is an important prerequisite for the success of chemical process industries. Tight time and cost constraints force these industries to continuously reduce their experimental effort during process development and to facilitate, even routinize, the application of model-based process technology. Nevertheless, the effort of setting up a detailed mathematical model for a chemical process remains still high because of the large variety of chemical process units and physical phenomena as well as increasing requirements on the sophistication of models. In order to overcome these limitations a systematization of modeling as well as the development of computer-aided modeling tools is required.

Recent publications of our group dealt mostly with the structuring of models and the introduction of canonical modeling objects (cf. left side of Fig. 1). Marquardt (1995) describes modeling objects for the structural and phenomenological description of chemical processes from a chemical engineering perspective. Other publications (cf. references cited in Marquardt, 1995) concentrate on the formal representation of modeling objects by means of the objectoriented data model VeDa. While these publications describe chemical process modeling from a so-called product-oriented point of view, this contribution will focus on an activity-or process-oriented point of view (cf. right side of Fig. 1), which takes account of the fact that a large part of chemical process modeling knowledge lies in the methodology and experience how models of chemical processes are set up. Thus, the emphasis will not be laid on the structuring of models but on the systematization of the modeling process, in particular from a chemical engineering perspective. The main goals of this contribution are to motivate the support of the modeling process, to discuss and illustrate important characteristics of the modeling process and to suggest a first set of canonical modeling steps allowing to classify and continuously extend procedural modeling knowledge.

Supporting the modeling process helps to overcome the modeling bottleneck described above for various reasons. Guiding modeling engineers by appropriate modeling process definitions, which provide information on the selection of modeling objects or of actions to be performed next, leads to an increased quality of models in terms of correctness, consistency, robustness or appropriate degree of detail. Since the quality of a model has to be 'produced' the modeling process executed during model development is responsible for the model quality (cf. Pohl, 1995).

Furthermore, the modeling process plays an important role in reducing model development times. E.g. the modeler can be relieved from routine tasks like the dimensional reduction of balance equations as described by Gerstlauer $e t$ al. (1993) if appropriate modeling steps have been defined. As often more than $50 \%$ of the model development time

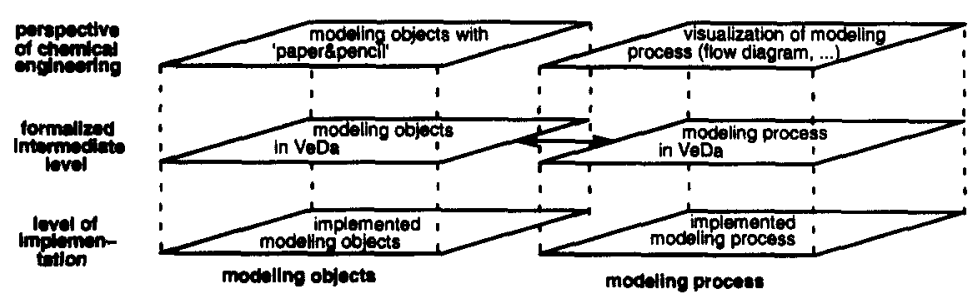

Fig. 1. Different views of chemical process modeling. is spent on searching for existing information, model development times can also be shortened by providing appropriate expert knowledge depending on the state of the model.

Another important issue in supporting model development is to improve model documentation in order to facilitate the reuse of models and to record the experience gained during model development. This includes automatic tracing of model- 
ing objects being selected, specified and aggregated, modeling steps being executed and modeling decisions including their rationale (cf. Bañares-Alcántara and Lababidi, 1995). This information can help to improve the modeling process (experience-based learning), which will in turn improve model quality, lead to shorter model development times etc. Finally, the modeling process itself can serve as a 'tutorial' for modeling engineers who want to improve their understanding of modeling and modeling methodology.

\section{THE MODELING PROCESS}

Since no detailed investigations into the methodology of setting up chemical process models are available in literature, this section will identify fundamental characteristics of the modeling process as a whole (Jarke and Marquardt, 1995). For this purpose the modeling process can be interpreted as a trajectory in a three-dimensional space spanned by the coordinates of specification, representation, and agreement (Fig. 2) in analogy to the representation of the requirements engineering process in software engineering (Pohl, 1995).

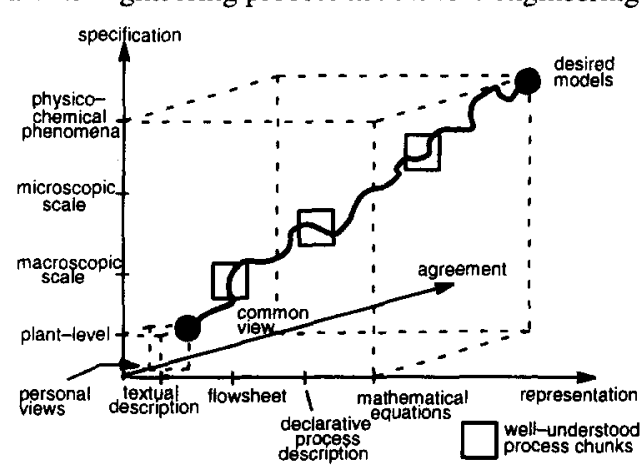

Fig. 2. Three dimensions of process modeling.

The specification dimension relates to the understanding of the model and to the concepts used for chemical process modeling on different levels of granularity. A coarse specification concentrates on the plant level and describes for example different sections of a plant. More detail is added on the process unit level. Macroscopic and microscopic scales of specification may be distinguished until all physico-chemical phenomena occurring in the chemical process are finally specified to the required degree of detail.

The representation dimension deals with different formalisms used to express knowledge of the system. Fig. 2 shows that in the early stages of chemical process modeling typically only informal natural language representations are used. Later on, semi-formal flowsheets and other schematic drawings are added. Formal languages (e.g. conceptual data models) should be employed to represent the chemical process specification explicitly and completely. Finally, the model is represented by

mathematical equations.

The agreement dimension captures the degree of agreement reached among different team members (modelers, experts for some unit operations, operating personal etc.) involved in a modeling project. Arguments in a certain context are employed to present the position of a team member, which forms the basis for subsequent conflict detection and resolution.

The modeling process can be visualized by a trajectory through this problem space. If the model is developed from scratch without using existing model elements this trajectory may start in the lower left corner of the cube (initial state) with a coarse understanding of the chemical process to be modeled, an informal representation and personal views of the modelers involved. The trajectory ends near the upper right corner of the cube (goal state) with a complete specification of the model for the chemical process, which is represented by a set of mathematical equations, and a set of agreed arguments explaining why the model looks the way it does. From the viewpoint of artificial intelligence, which describes problem solving as a movement in a state space (cf. Winston, 1993), the path from the initial to the goal state can be planned if all the activities are properly understood. This is, however, not true for process modeling. Planning is hence only possible in part for well-understood process chunks (cf. Fig. 2).

\section{MODELING STEPS}

In order to reduce complexity it is reasonable to decompose the modeling process into so-called modeling steps. These can te defined as activities (i.e. information retrieval, decisions, actions etc.) leading to a modification of the state of a model. Modeling steps might be composite, if they can be decomposed into more refined modeling steps (substeps), or elementary, if they are not decomposable. Both elementary and composite modeling steps should be independent from a specific modeling problem. Once having been defined for a specific modeling project and generalized subsequently - modeling steps can be reused in following projects just like modeling objects. The attributes listed in Table 1, which characterize elementary and composite modeling steps in general, will be discussed in the following. It should be noted that these attributes serve to discuss the properties of modeling steps and not to describe possible formalizations.

Name. Each modeling step should have an unambiguous name.

Goal. This attribute states the outcome ('goal') towards which the modeling step is directed. Hence, the purpose of a modeling step consists in achieving this goal (goal achievement, cf. Rosenbloom, 1993). Two modeling steps differing only in the goal attribute must lead to different results.

Pre- and postconditions. Preconditions determine which subset of all modeling steps can be enacted (executed) at a certain point on the trajectory depicted in Fig. 2. These conditions check for example whether all modeling objects needed for enacting a modeling step exist and whether these modeling objects are correctly defined. E.g. enacting the modeling step 'define reaction kinetics' requires that the reacting chemical species have been defined. Postconditions check whether the result of the modeling step corresponds to the desired result, i.e. whether the goal of the modeling step has been achieved. Preconditions are enforced during the enactment of the modeling process. As a consequence, only those preconditions should be defined which are absolutely necessary for consistency and correctness reasons in order not to restrict the creativity of the modeling engineer.

Schedule. Like pre- and postconditions a schedule captures knowledge of sequencing of modeling steps. But while pre- and postconditions restrict the number of possible sequences of substeps, the schedule guides modeling engineers by proposing one sequence of modeling steps. Consequently, the schedule defines in well-understood process chunks a plan in the sense of planning (cf. Winston, 1993) to achieve the goal of a composite step. This plan is not enforced, so that deviations from the suggested solution path are possible. By changing the schedule consistently with the pre- 
conditions alternative composite modeling steps can be predefined capturing alternative strategies (plans). In addition to proposing a strategy, this attribute serves to trace (document) the strategy chosen by a modeler during the enactment of the composite modeling step.

Table 1. Attributes characterizing modeling steps.

\begin{tabular}{|l|l|l|}
\hline attribute & $\begin{array}{l}\text { explanation of attribute for an elementary } \\
\text { modeling step }\end{array}$ & $\begin{array}{l}\text { explanation of attribute for a composite mo- } \\
\text { deling step }\end{array}$ \\
\hline \hline name & name of modeling step \\
\hline goal & states the outcome ('goal') towards which the modeling step is directed \\
\hline preconditions & all conditions to be satisfied before the modeling step is termed enactable \\
\hline postconditions & all conditions to be satisfied before the modeling step is termed terminated \\
\hline schedule & - & specifies one possible sequence of substeps \\
\hline modeling objects & all modeling objects the modeling step is applicable to \\
\hline status & enactable, not enactable, enacting, terminated, aborted \\
\hline actions & $\begin{array}{l}\text { everything to be done in order to achieve the } \\
\text { goal of an elementary modeling step }\end{array}$ & - \\
\hline substeps & - & all substeps of composite modeling steps \\
\hline decisions & decisions to be made during enactment of the modeling step \\
\hline
\end{tabular}

Modeling objects. Modeling objects like process quantities or model equations are an important part of the description of the states in the problem space spanned by the three dimensions of process modeling (Fig. 2). Since a modeling step leads from one state (input state) to another one (output state), there is a close relationship between modeling steps and modeling objects, which can be subdivided into input and output modeling objects. In order to be sure that there is a movement into the direction of the goal state during the execution of the modeling process, i.e. more and more information on the model is specified, the output modeling objects should be specializations of the input objects. Obviously, two different modeling steps can have the same input state, if one path splits in two alternative ones, or the same output state, if two paths join.

Relying solely on modeling objects without being supported by a modeling process would require the modeler to find a path from the initial to the goal state on his own without knowing if there is a better one and without being guided during model development. Modeling steps leading from one state to another provide the knowledge whether the movement from one specific state to another one is possible due to pre- and postconditions or recommended due to the schedule. Hence, the search space of all possible paths is reduced and the modeler is guided to find a path a real expert in modeling would take.

Status. Depending on pre-and postconditions as well as on the schedule the status of a modeling step might change. If the preconditions as well as conditions specified by the schedule are satisfied, the status becomes 'enactable'. If a step is enactable and the modeling engineer has decided to execute the step, the status changes to 'enacting'. Depending on the postconditions the status is set to 'terminated'. If a modeling step has been enacted and not been terminated and the modeler has decided to do something else, the status changes to 'aborted'.

Actions. This attribute describes everything that has to be done in order to achieve the goal of an elementary modeling step. According to the degree of formality of this attribute different types of modeling steps can be distinguished. Examples are automatic modeling steps like the symbolical manipulation of model equations, which can be executed in an algorithmic form, or manual modeling steps like writing down a new model equation requiring at least partly the knowledge of the modeling engineer.

Substeps. The introduction of composite modeling steps, which are by definition decomposed into more refined modeling steps (substeps), leads to a composition hierarchy of modeling steps. Since every modeling step is related to a goal, this hierarchy of modeling steps is paralleled by a hierarchy of goals.

Decisions. During the execution of elementary and composite modeling steps many decisions have to be made, so that supporting decision making and tracing decisions including their rationale is an important prerequisite for the support of the modeling process and its experience-based improvement. Two kinds of decision models, Multiple Attribute Decision Making (MADM) and the Issue-based Information Systems model (IBIS), are considered for supporting the process of decision making. MADM, which was developed in decision theory, describes a decision by four components (cf. Zimmermann and Gutsche, 1991): The first component describes a set of alternatives, from which one alternative has to be selected. The second component consists in the set of criteria, against which the alternatives have to be evaluated. The third one corresponds to the set of ratings (performance) of each alternative with regard to each criterion, and the fourth one to a set of value functions, which describe - based on the ratings - the preferences of the persons involved in the decision. If for example the modeling problem in Fig. 4 is considered, a possible decision might concern the question whether heat exchange between the fluid and the environment should be taken into account (first alternative) or not (second alternative). Two possible criteria for the evaluation of the alternatives might be the degree of detail of the model and the modeling effort required. As for most decisions to be made during modeling, the ratings for each alternative can only be expressed by fuzzy verbal expressions like 'the modeling effort for the first alternative is quite high'. Consequently, setting up value functions is very difficult and time consuming. More appropriate with regard to this problem is the IBIS model, which was developed in design theory. According to this decision model each alternative (position) for solving a decision problem (issue) is evaluated on the basis of argumentation (arguments), which is particularly useful for group decisions (cf. Conclin and Begeman (1995) for more detail and Jarke and Marquardt (1995) for an example of a decision made during chemical process model development).

The complex relation between steps, substeps, goals and decisions, which has not been treated in appropriate depth in literature so far, can be illustrated by use of Fig. 3. If the material balance equation (cf. Fig. 4) is given, a goal G0 called 'specification of balance equation by additional equations' can be set, which corresponds to the goal attribute 
of modeling step M0. The complexity of the problem can be reduced by defining subgoals G1,... G3 concerning the specification of each term of the mass balance equation (goal decomposition). Hence, achievement of goals G1 to G3 leads to the achievement of goal G0 (cf. Rosenbloom, 1993). These goals correspond again to the goal attribute of modeling steps (M1, .. M3, which are substeps of modeling step M0). Regarding the mass transfer rate (goal G3) different alternatives might exist for its specification. As a consequence, goal G3 corresponds to a set of alternative goals G3.1, ... G3.3, each corresponding to one alternative law describing the mass transfer. Consequently, there are also three corresponding modeling steps M3.1, ... M3.3. Since these goals (modeling steps) are alternative ones, a decision concerning the selection of one goal (modeling step) has to be made (goal refinement, cf. Pohl, 1995). Goal decomposition of goal G3.1 leads again to a further specification of the process quantities of the transfer law.
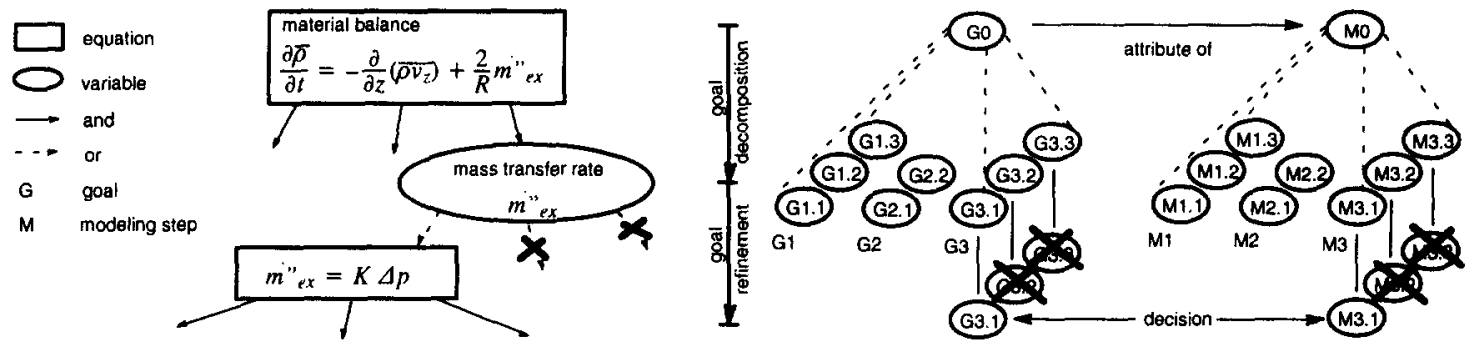

Fig. 3. Hierarchical refinement of a balance equation.

\section{AGGREGATION OF MODELING STEPS}

Before addressing a new modeling problem a new modeling process to be enacted during model development has to be aggregated from modeling steps, or at least an existing modeling process has to be adapted. Aggregation of modeling steps corresponds to defining a complete hierarchy of composite and elementary modeling steps, such that the composite step at the top of this hierarchy corresponds to the complete modeling process. Furthermore, aggregation includes the proposition of a strategy that will be followed during model development by defining schedules for enacting modeling steps. In order to make sure that modeling engineers enacting the modeling process acquire expert knowledge concerning the methodology of setting up chemical process models, the modeling process to be enacted during model development should only be prepared by real experts of modeling. Two general alternative strategies of model development which can be prepared by such modeling experts are the top-down- and the bottom-up-approach. With regard to the behavioral description of the porous tube depicted in Fig. 4 the top-down-approach starts with the material balance equation and consecutively refines its terms as depicted in Fig. 3. The bottom-up-approach would start at the bottom of the hierarchy outlined on the left of Fig. 3 specifying for example the modeling equation for the mass transfer coefficient first. Usually, a mixture of both approaches is applied while setting up the modeling process.

Based on the modeling example in Fig. 4 this section will describe the modeling process in a top-down-manner in the following. The modeling process will be represented by a directed graph with nodes corresponding to modeling steps and edges describing the scheduling of steps (cf. Fig. 5).

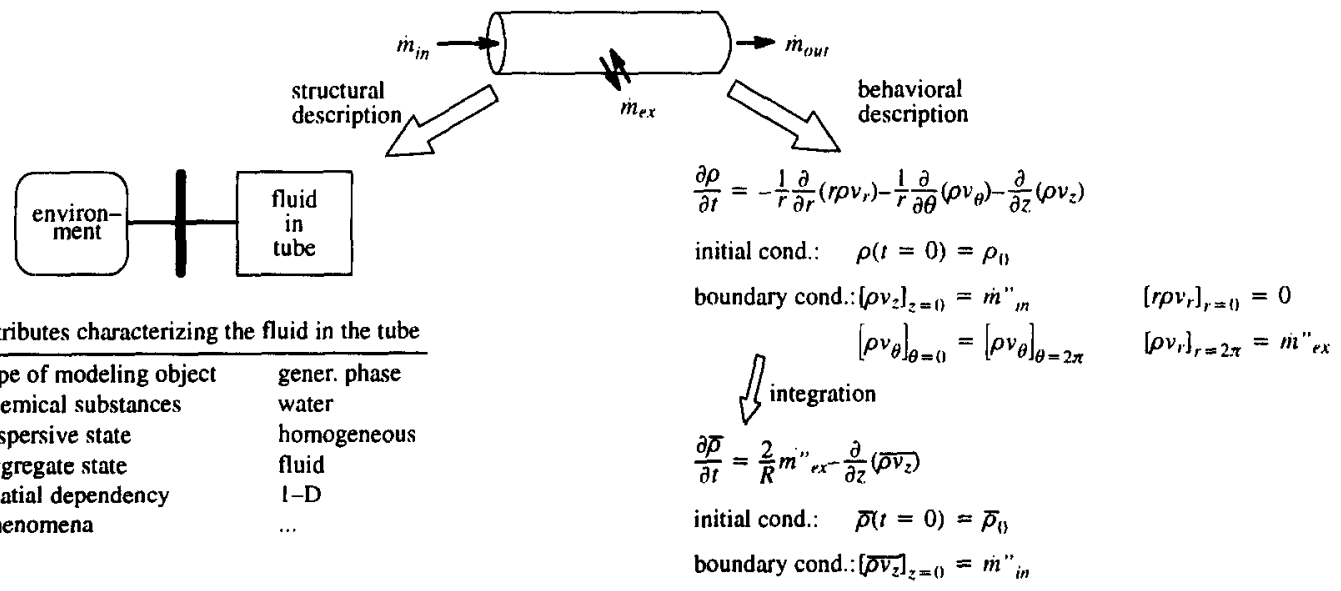

Fig. 4. Structural and behavioral description of a porous tube (cf. Marquardt, 1995).

Specify Requirements. In a first step the modeling engineer should always write down some requirements on the model to be set up, e.g. the goal of the model, the desired degree of detail or major assumptions. It should be possible to enact this modeling step again during the whole rest of the modeling process, as normally not all requirements are known at the beginning of model development. For reasons of simplicity this has been neglected in Fig. 5. Right at the beginning there are no relations between this modeling step and any modeling objects, since no modeling objects have been created yet. To write down requirements on a model is a very creative step which cannot be formalized. The only support for a modeling engineer might be a classification scheme for different kinds of requirements. As a 
consequence, this step is a manual modeling step. With regard to the modeling example in Fig. 4 a possible requirement might concern the neglection of the energy exchanged between tube and environment via the porous wall.

Define model structure. After having set up a preliminary set of requirements the structure of the chemical process to be modeled has to be described (cf. left side of Fig. 4). This structure is modeled by two different classes of structural modeling objects, namely devices and connections, which are distinguished according to the functionality assigned to them during the modeling process (Marquardt, 1995). The role of a device is the transformation of its internal state variables according to known sources and fluxes acting on the device from its environment. A connection provides the fluxes to the adjacent devices according to the driving forces determined by the states of the adjacent devices. In contrast to a device, a connection never stores any extensive quantity. Devices and connections are termed composite, if they form aggregates of devices and connections, and elementary, if they are not decomposable in a certain modeling context.

The modeling step 'define model structure' is a composite step consisting of the substeps depicted in Fig. 5. The first substep deals with the selection of a delimitable material part of the chemical process to be represented by a structural modeling object. Obviously, this selection depends on the modeling context and cannot be formalized. Only some heuristic rules can serve to guide the modeler. During the enactment of the modeling process of the porous tube for example the fluid in the pipe was selected as a material entity.

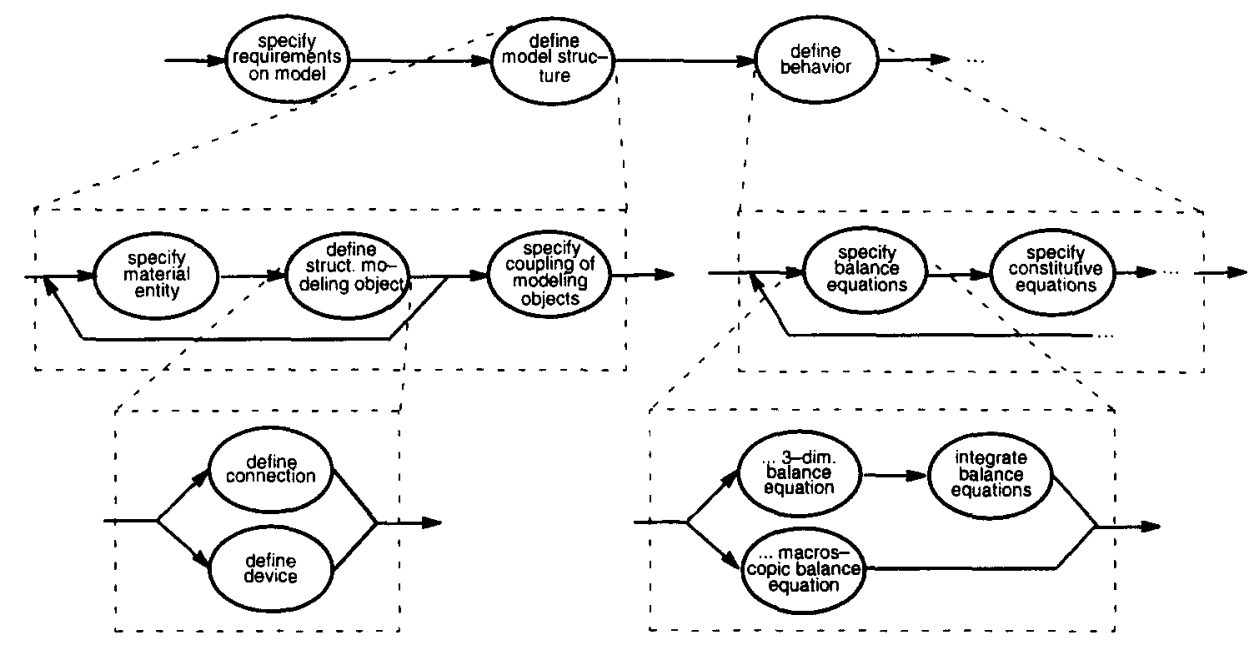

Fig. 5. Aggregation of modeling steps.

The following modeling step 'define structural modeling object' requires as a precondition that a material entity has been selected. The goal of this step is to abstract the material entity by a modeling object type such as a device or a connection and their various specializations including a complete specification of the characterizing attributes (cf. Fig. 4). As depicted in Fig. 5 this modeling step is a composite one comprising two substeps and a decision about the choice between a device and a connection. The modeling steps 'define device' and 'define connection' are again composite steps comprising decisions concerning different types of devices and connections as well as substeps for a further specification of characterizing attributes. For the decisions concerning the selection of the appropriate type of modeling object the modeling process can automatically provide the alternatives including heuristic rules supporting the selection.

In Fig. 5 an iteration loop is depicted after the step 'define structural modeling object', since always more than one material entity has to be considered on a hierarchical level of structural description. After on one hierarchical level all structural modeling objects have been introduced and completely defined, those connections and devices have to be coupled which exchange signals or information on fluxes and states ('specify coupling of modeling objects'). It should be noted that the aggregation and sequencing of these modeling steps for the structural description is not unique. It would be reasonable to define the coupling between some structural modeling objects before all of them have been completely defined. As described above, to change the sequence of modeling steps means to change the schedule by enacting the meta-modeling process.

Define behavior. After setting up the model structure the behavior of the modeling objects has to be described. If a top-down-strategy is applied the modeler starts with the balance equations and refines their terms (source term, exchange term, etc.) afterwards by writing down additional constitutive equations (cf. above).

The precondition of the step 'specify balance equations' is that a corresponding structural modeling object exists and is completely defined, i.e. all characterizing attributes are specified. The postcondition has to check, whether the balance equations are correct. Obviously, it is impossible to completely formalize this modeling step, but based on the specifications of the characterizing attributes (spatial dependency, phenomena, ...) 'raw' balance equations can be generated automatically, which have to be specified afterwards.

As depicted in Fig. 5 this modeling step comprises three modeling steps as well as a decision concerning the spatial dependence of the balance equations. If spatial dependence is to be considered it is reasonable to start with balance equations in three dimensions including initial and boundary conditions. In Fig. 4 a material balance equation in three dimensions for the fluid in the tube is depicted. Applying the modeling step 'integrate balance equations' leads to the desired balance equations. As described by Gerstlauer et al. (1993) this modeling step is an automatic one, which can be completely formalized. The result of applying this step is also shown in Fig. 4. 
Just as the aggregation of modeling steps for the structural description the one for the behavioral description is not unique. Instead of writing down all balance equations for a structural modeling object before refining the terms by additional equations, some modelers might prefer to write down and refine one balance equation before taking another one. Hence, the schedule of modeling steps might change from modeler to modeler or from modeling project to modeling project. A number of reasonable alternatives can however be captured by means of a composite modeling step.

Due to the complexity and variety of chemical process units and physico-chemical phenomena a large number of modeling steps is necessary in order to support modeling in every detail. Of course, it is impossible to develop a complete framework of modeling steps at once. However, the following definition of a first set of canonical modeling steps can serve as a classification scheme to be extended continuously in the future by additional steps, which proved to be useful in modeling projects.

Obviously, the definition of such a classification scheme is not unique. The classification scheme represented in Fig. 6 organizes the modeling steps depicted in Fig. 5 in a specialization tree, i.e. the modeling objects being modified or aggregated by a modeling step located on a lower branch of the tree are specializations of those being treated by a modeling step on an upper branch.

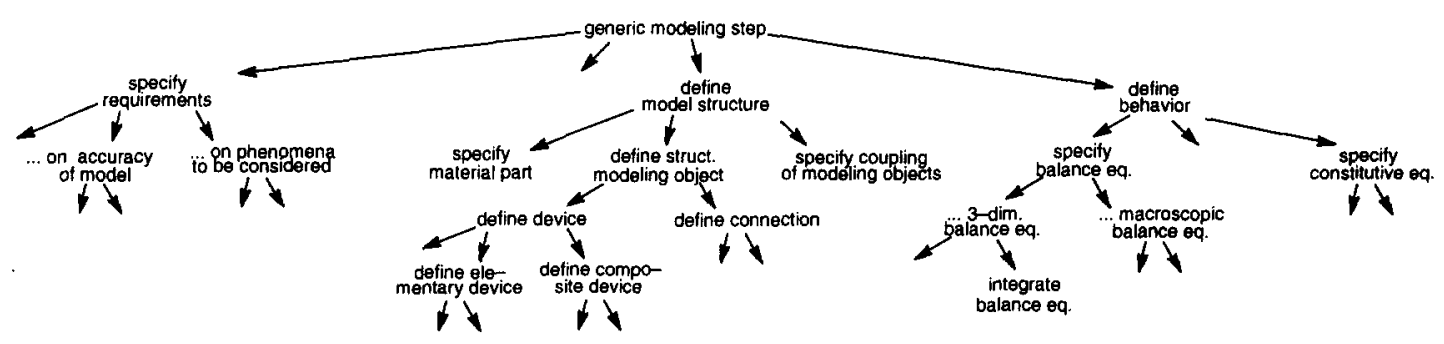

Fig. 6. Classification scheme of modeling steps.

\section{CONCLUSIONS}

This paper shows that in addition to the knowledge about models, a modeling methodology should comprise knowledge about the modeling process in order to effectively support the development of new and the adaption of existing models for chemical processes. General properties of the modeling process and of modeling steps are discussed and jllustrated. Furthermore, a first set of canonical modeling steps allowing to classify and continuously extend procedural modeling knowledge is proposed. In order to integrate the support of the modeling process into existing modeling technology, further work has to be conducted into four directions: First of all, a larger set of canonical modeling steps has to be determined and ultimately formalized using the object-oriented data model VeDa (cf. Marquardt, 1995). Secondly, useful strategies of modeling have to be identified, which can be captured by specifying the schedule attribute of composite modeling steps. Furthermore, formalisms like Petri Nets or constraint languages controlling the enactment of processes have to be selected and adapted. Finally, all concepts necessary for supporting the modeling process have to be implemented by use of an appropriate knowledge representation language. This includes the implementation of automatic tracing mechanisms, in order to support the experience-based improvement of the modeling process.

\section{ACKNOWLEDGEMENTS}

The authors appreciate the numerous fruitful discussions with M. Baumeister, R. Bogusch, R. Dömges, M. Jarke and K. Pohl as well as the financial support of the DFG ('Deutsche Forschungsgemeinschaft').

\section{REFERENCES}

Bañares-Alcántara, R. and Lababidi, H.M.S., 1995, Design support systems for process engineering I and II, Computers chem. Engng 19, pp. 267-301.

Conklin, J. and Begeman, M.L., 1988, gIBIS - a hypertext tool for exploratory policy discussion, ACM Trans. Office Information Systems, pp. 303-331.

Gerstlauer, A., Hierlemann, M. and Marquardt, W., 1993, On the representation of balance equations in a knowledgebased process modeling tool, CHISA'93, Prague, Czech Republic.

Jarke, M. and Marquardt, W., 1995, Design and evaluation of computer-aided process modeling tools, Proc. Intelligent Systems in Process Engng. ISPE'95, July 1995, Snowmass, USA.

Marquardt, W.,1995. Trends in computer-aided process modeling. Accepted for publication in Comput. Chem. Engg.

Pohl, K., 1995, A process centered requirements engineering environment, Doctoral dissertation, Lehrstuhl für Informatik V, RWTH Aachen University of Technology, Aachen.

Rosenbloom, P.S., 1993, A symbolic goal-oriented perspective on connectionism and soar, in: P.S. Rosenbloom, J.E. Laird and A. Newell (ed.): The Soar Papers Volume II, pp. 821-839, MIT Press, Cambridge, 1993.

Winston, P.H., 1993, Artificial intelligence, 3rd Edn, pp. 323 ff., Addison-Wesley, New York.

Zimmermann, H.J. and Gutsche, L., 1991, Multi-Criteria Analyse - Einführung in die Theorie bei Mehrfachzielsetzungen, Springer, Berlin. 